\title{
The Component Data for E-portfolio
}

\author{
Yanxia Pang ${ }^{1, a}$, Jumei Zou ${ }^{2, b}$, Shaochan Dong ${ }^{3, C}$ \\ ${ }^{1}$ Academy of Computer and information, Shanghai Second Polytechnic University, Shanghai, \\ 201209, China \\ ${ }^{2}$ Department of Education Information Technology, Hangzhou Normal University, Hangzhou, \\ 310018, China \\ ${ }^{3}$ Cloud Computing and Big Data Center, Academy of Software, East China Normal University \\ Shanghai, 200333, China \\ aemail: ladypang@163.com, bemail: jumeiz@163.com, cemail: scdong510@163.com
}

Keywords: E-portfolio; component; data; xsd; standard; evaluation

\begin{abstract}
We introduce the component data for E-portfolio(Electronic portfolio). E-portfolio) is used to cllect works of students and aimed at learning evaluation, so the components of E-portfolio must be standardized. We do some research on the component data structure of E-portfolio. Based on the features of E-portfolio we use xml file as the storage format of E-portfolio. Thus we can define xsd file which is the structure file of $\mathrm{xml}$ file for the users. People can build their E-portfolio on the structure xsd file. In this way E-portfolio based on xsd structure are in same format. That helps for further E-portfolio share and evaluation.
\end{abstract}

\section{Introduction}

Portfolio's meaning is similar to file folder. In the field of educational technology E-portfolio( electronic portfolio) records the process of learning with a variety of data for many users including students and teachers. But it is different from a file folder. First data of electronic portfolio may be different forms of media such as text, audio, video, etc. Second it includes two kinds of feedback. One is evaluation by teachers and other students, the other is self evaluation. Users need to be aware of these feedback, knowing the problem that they illustrate to guide their practice. Finally in the digital era it is more in electronic format, in this paper we call the electronic format learning file as E-portfolio.

E-portfolio documents the user's learning data in the learning process and the final learning result at the end. According to these data, we can use the traditional approach for not only summary evaluation but also formative assessment. In this way we can evaluate learners in the learning process. The learners can be stimulated to learn better. This is quite different from the current examination system in a test set. Diy E-portfolios can also stimulate the user's enthusiasm and creativity. Learners can creat their own portfolio to show what they learn. To participate in the evaluation also stimulate their learning initiative. But many learners don't even know what is E-portfolio. And E-portfolioes by different users have different content. It's hard to compare them for evaluation. Here we try to give a good way to give a frame for E-portfolio. So learners can make it clear what components a piece of E-portfolio of his kind should include and in which kind of sequence. The following parts first analyze E-portfolio's components and give an idea of E-portfolio's data storage mode with xml files.

\section{Components of E-portfolio}

With the trend of further division in industry, many areas have divided their work into more parts. For example, more and more software is developed with components. Components here mean some units reused usually, so people make them into packages called objects. In this way, people do not have to concern the code but to care more about the components' function and how to use them. 
And the engineers just need to write the code and package them for one time. After that people reuse it any times they want. They call the package with parameters value and the answer they want as componnet. Component as an idea of instruction was proposed by Merrill decades ago. E-portfolio is a collection of a student's work, used for learning performance share and evaluation . Here we try to use component for E-portfolio in application. Because componentization has such advantages as resource reusing, modeling for same kind problem with individual results, we mean to give the portfolio users some help with componentization of E-portfolio.

Electronic portfolio is mainly a collection about learning, usually used for learning share and evaluation. Nowadays E-portfolio is used for more kinds of learning. But the application of E-portfolio shows some difficulties. People make E-portfolio again and again for different purpose. That like what happened about software developing before. We need a new idea for resource reuse and lower cost.

Componentization of E-portfolio means E-portfolio departed into components. Thus E-portfolio becomes more reusable and easily rearranged.

Components in E-portfolio are information of the E-portfolio it belongs to. It is mainly learning data including learning goal, learning works, evaluation marks, etc. It includes not only code or class but also multimedia learning data. The multimedia data can be different kinds of media such as video, audio, text, image, etc.

\section{Data of Componentized E-portfolio}

E-portfolio is mainly a collection of learners' work. A piece of E-portfolio for better evaluation must includes at least components of portfolio description and content. The portfolio description gives the basic information of this E-portfolio such as time, author, title, etc. The content includes mainly the learning works to show the learning. On this point, we find xml fits the component idea very well. And it can store its structure in xsd file. The xml files with same xsd file must have same structure according the content of the xsd file. Once we give the xsd file to the users, they just need fill the xml values. This feature fits the standardization of E-portfolio for evaluation and helps the users to build E-portfolio more easily.

A. E-portfolio Description Component

E-portfolio Description Component is about the basic information of E-portfolioes. To make the attribtes of different E-portfolioes in same structure we give a xsd file to define the basic attributes of E-portfolio. Every portfolioe maker will have to supply the meta-data of the E-portfolio like name, time according to the standards of E-portfolio made by CELTSC (China E-Learning Technology Standards Comminitte). Here we give part of the XSD file as an example.

$<$ xs:element name="portfolio" $>$

$<$ xs:annotation $>$

$<$ xs:documentation $>$ Comment describing your root element $</$ xs:documentation $>$

$</$ xs:annotation $>$

$<$ xs:complexType $><$ xs:sequence $><$ xs:element ref="general" $/>$

$<$ xs:element ref="life_cycle"/ $><$ xs:element ref="meta_metadata"/ $>$

$<$ xs:element ref="technical"/> <xs:element ref="education"/>

$\ldots$

The XSD file constructs the meta-data of E-portfolio by the elements . Users can give the information about their E-portfolioes according to the XSD files. And every elements has their data type. XSD file will verify the value users fill in. In this way meta-data standards get into the E-portfolioes. They do not have to read about the standards and just make xml files by the xsd structure.

B. Components for content

After the meta-data of the E-portfolioes, we come to the main body of E-portfolio which includes the learning information of the user. Here we work on the summarization E-portfolio as an example. Figure 1 shows the Concept design of summarization E-portfolio. E-portfolio of 
this kind is made mainly for final evaluation. It must give the eric and the original goal for testing. In the end we ask the learner give a new goal for further learning. So summarization E-portfolio includes goal, work collection, self-evaluation, metric and new goal, etc. Part of the XSD file for elements structure definition is listed as following:

...

$<$ xs:element name="summarization E-portfolio">

$<$ xs:annotation $>$

$<$ xs:documentation>Comment describing your root element </xs:documentation>

$</$ xs:annotation $>$

$<$ xs:complexType $>$

$<$ xs:sequence $>$

$<$ xs:element name="goal" type="file"/>

$<$ xs:element name="works passed" $><$ xs:complexType $><$ xs:sequence $>$

$<$ xs:element name="works" type="file" minOccurs="0" maxOccurs="unbounded"/>

$</$ xs:sequence $><$ /xs:complexType $><$ /xs:element $>$

$<$ xs:element name $=$ "works unpassed" $><$ xs:complexType $><$ xs:sequence $>$

$<$ xs:element name="works" type="file" minOccurs="0" maxOccurs="unbounded"/>

$</$ xs:sequence $></$ xs:complexType $></$ xs:element $>$

$<$ xs:element name="metric" type="file"/ $>$

$<$ xs:element name="self evaluation" type="file"/><xs:element name="new goal" type="file"/>

$</$ xs:sequence $><$ /xs:complexType $></$ xs:element $></$ xs:schema $>$

The components of summarization E-portfolio includes goal, works passed, works unpassed, metric, self evaluation and new goal. Works passed means works made by learners that meet the requirement of metric. Then works unpassed means works which is not good according to the metric. The attributes minOccurs means the minimum amount of the element. And maxOccurs means the maximum amount of the elment.

Figure 2 is about the XSD file structure of the summarization portfolio. By this figure, we can make a better view of the above xml file. XSD file is the structure file of xml. It makes all the E-portfolio saved as xml file in same format. They all must include the element xsd required. And all the elements must obey the format attributes XSD file gives. Some components which have the "minOccur" attribute as 0 do not have to be included in the E-portfolio.

C. Leaf Component

Leaf component is component that can not be divided any more. Most leaf components are file type which must supply file name and file description. File type defines mainly the works learner makes in their learning process. File type is defined in XSD file as following:

$<$ xs:complexType name="file" $>$

$<$ xs:sequence $>$

$<$ xs:element name="filename" type="xs:string"/>

$<$ xs:element name="annotation" type="xs:string"/>

$</$ xs:sequence $>$

$</$ xs:complexType $>$

Here we can see the leaf component's format which are designed in the same frame. The filename must be defined as string type. So does the annotation. They are showed in the above sequence that means filename first and annotation second. All of this is for the standardization of different E-portfolioes. The goal of standardization is faced for better learning works share and evaluation.

\section{Conclusion}

In this text we gave XSD as a way to give component structures of E-portfolio. It provides the components that E-portfolio must include. That makes E-portfolio made more easily. The E-portfolio is in almost completely same forms which can be used for evaluation with other E-portfolioes in same forms. Data storage in xml format fits the E-portfolio quite well. It helps the 
users with a clear portfolio frame, and makes the learning share and evaluation between different E-portfolioes more easily.

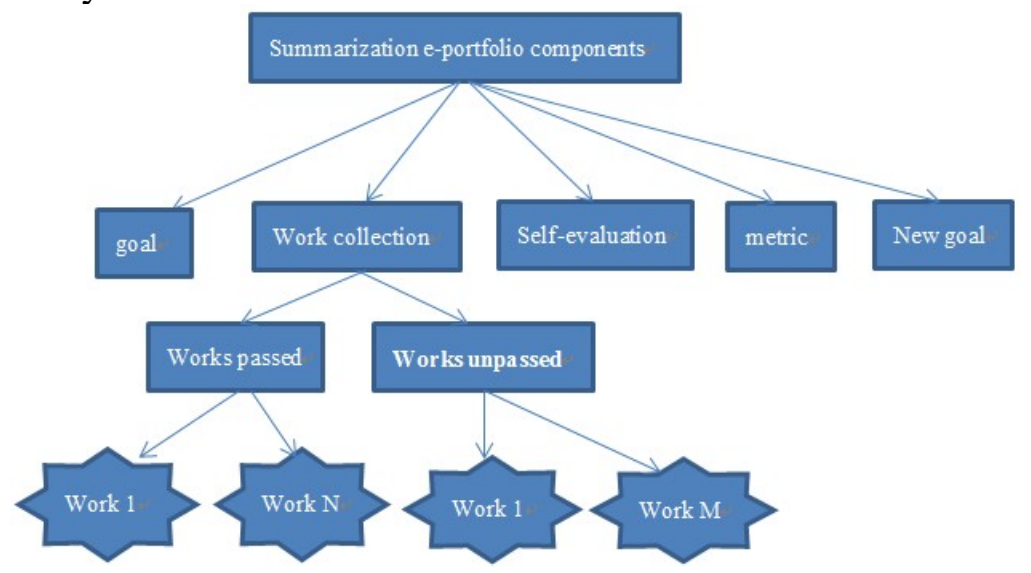

Fig.1. Component Structure of Summarization E-portfolio

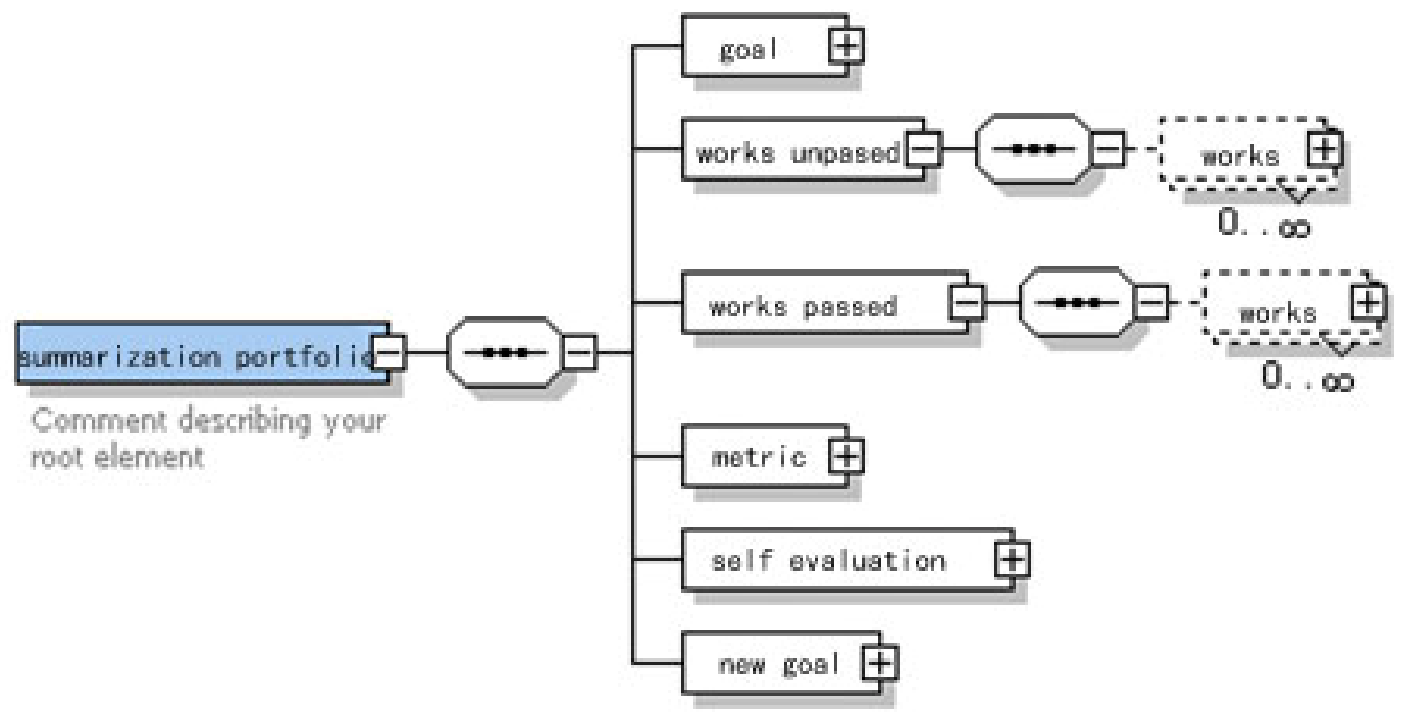

Fig.2. XSD file structure of summarization portfolio

\section{References}

[1] Helen C. Barrett: Researching the Process and Outcomes of Electronic Portfolio Development in a Teachers Education Program, SITE (International Society for Technology in Education), 2003.

[2] M.D. Merrill: Component Display Theory, http://tip.psychology.org/merrill.html, 01/05/2004.

[3] Helen C. Barrett, Competing Paradigms in Electronic Portfolio Approaches, http://electronicportfolios.org/systems/paradigms.html,01/02/2004.

[4] Barrett, H. Frequently-asked questions about electronic portfolios. Retrieved July 17, 2008, from http://electronicportfolios.org/faq.html, 2008

[5] Bratengeyer, E. The advent of eportfolios in Europe. International Journal of Emerging Technologies in Learning, 3(1), 4-5,2008.

[6] Attwell, G.. Personal Learning Environments - the future of eLearning? eLearning Papers, (vol. 2 no. 1), p.1 - 8 , 2007.

[7] Dino Esposito, Applied Programming for Microsoft.NET, China Machine Press, 2003. 